\title{
Effects of Conditioning Stimulation of the Central Amygdaloid Nucleus on Tooth Pulp-Driven Neurons in the Cat Somatosensory Cortex (SI)
}

\author{
Kei KAWARADA, Ken-ichi KAMATA, and Norio MATSUMOTO \\ Department of Oral Physiology, School of Dentistry, Iwate Medical University, \\ Morioka, 020-8505 Japan
}

\begin{abstract}
To study the limbic control of nociception, we examined the effect of conditioning stimulation of the central amygdaloid nucleus (ACE) on tooth pulp-driven (TPD) neurons in the first somatosensory cortex (SI). Cats were anesthetized with $\mathrm{N}_{2} \mathrm{O}-\mathrm{O}_{2}(2: 1)$ and $0.5 \%$ halothane, and immobilized with tubocurarine chloride. The tooth pulp test stimulus was applied by a single rectangular pulse $(0.5 \mathrm{~ms}$ in duration and $3-5$ times the threshold intensity for the jaw-opening reflex). Conditioning stimuli to the ACE consisted of trains of 33 pulses $(300 \mu \mathrm{A})$ delivered at $330 \mathrm{~Hz}$ at intervals of $8-10 \mathrm{~s}$. In 35 out of 61 of the slow (S)-type TPD neurons with latencies of more than $20 \mathrm{~ms}$, conditioning stimulation in the ACE, especially in the medial division, markedly reduced the firing response to the pulpal stimulation. The inhibition of the firing rate in the S-type neurons was $74 \%$ of the control. In these S-type neurons, the neurons that were inhibited had sig-
\end{abstract}

nificantly longer latencies compared to the noninhibited neurons $(45.0 \pm 17.6 \mathrm{~ms}, n=32$ vs. $34.8 \pm 10.5 \mathrm{~ms}, n=26)$. In contrast, the ACE conditioning stimulation affected only one out of 18 fast-type TPD neurons with latencies of less than $20 \mathrm{~ms}$. In addition, ACE stimulation had no effect on the spontaneous discharges of either S-type or F-type neurons. The ACE inhibitory effect on S-type neurons was not diminished by naloxone administration (1 mg/kg, I.V.), while the blockade of histamine $\mathrm{H}_{1}$-receptor by diphenhydramine hydrochloride $(0.5 \mathrm{mg} / \mathrm{kg}$, I.V.) partially reversed the inhibitory effect. These results suggest that the ACE inhibits ascending nociceptive information to the $\mathrm{SI}$ and that this inhibition is mediated in part by histamine $\left(\mathrm{H}_{1}\right)$ receptors. It seems likely that the antinociceptive effect is a neurophysiological basis for stress-induced analgesia (SIA). [Japanese Journal of Physiology, 49, 485-497, 1999]

Key words: central amygdaloid nucleus, stress-induced antinociception, somatosensory cortex, tooth pulp-driven neuron, $\mathrm{H}_{1}$ receptor.

The sensation we call pain, like other sensations, can be modulated by exposure to a stressful situation such as a fight or athletic competition. It has been suggested that stress is a physiologic trigger of the intrinsic pain inhibitory system. This phenomenon has been termed as stress-induced analgesia (SIA) (see Amit and Galina [1] for review) or fear-induced analgesia [2]. Actually, acupuncture and transcutaneous electrical nerve stimulation, which might be considered stressful, have been used for analgesia as a clinical procedure [1]. However, the neuronal mechanism and anatomical substrates involved in SIA have remained unclear.

It has been demonstrated that the central amygdaloid nucleus (ACE) has an integrative role for coordinating an organism's autonomic, endocrine, and behavioral responses to various stressful stimuli, and that electrical stimulation of the ACE can mimic several of the stress-like responses (see Glavin et al. [3] for review). Lesions of the ACE have been found to block cardiovascular, respiratory, and behavioral parameters of responses to stressors [4, 5]. Intracere- 
broventricular infusion of corticotropin-releasing factor (CRF), which participates in a number of responses to stress or exposure to restraint stress, induces $c$-fos expression in the ACE [6]. Moreover, the microinjection of neurotensin or an enkephalinase inhibitor into the ACE has increased the nociceptive threshold in behavioral studies [7,8]. Therefore, it may be reasonably assumed that the ACE is concerned with SIA.

In order to confirm this assumption, the present study was performed to investigate whether ACE conditioning stimulation has an inhibitory effect on nociception. The firing activity of tooth pulp-driven (TPD) neurons in the oro-facial area of the first somatosensory cortex (SI) was used as an index of nociception. These TPD neurons have been classified into 3 types depending on their firing responses to tooth pulp stimulation: fast (F)-type neurons with a short latency of less than $20 \mathrm{~ms}$, slow (S)-type neurons with a long latency of more than $20 \mathrm{~ms}$, and Fa-type neurons with the responses of F-type accompanied by afterdischarges [9]. We report here that ACE conditioning stimulation inhibits the firing rate in S-type TPD neurons with a relatively long latency and that this inhibitory effect is reversed by an $\mathrm{H}_{1}$-antagonist but not an opioid antagonist. The present report is the first to offer electrophysiological evidence that the amygdala exerts profound inhibition on nociception. A brief preliminary report of these findings has been presented previously [10].

\section{METHODS}

Preparation of animals. Experiments were conducted on 54 adult cats weighing $1.9-4.7 \mathrm{~kg}$. For surgery, the animals were initially anesthetized with ketamine hydrochloride $(30 \mathrm{mg} / \mathrm{kg})$ and treated with atropine sulfate $(0.1 \mathrm{mg} / \mathrm{kg}$, I.P.). The femoral vein was then cannulated for perfusion and the administration of drugs, and a tracheotomy was carried out for artificial respiration. Animals were immobilized by intravenous perfusion of tubocurarine chloride $(1.4 \mathrm{mg} /$ $\mathrm{kg} / \mathrm{h}$ ) and artificially ventilated with a gaseous mixture of $\mathrm{N}_{2} \mathrm{O}-\mathrm{O}_{2}(2: 1)$ and $1.5 \%$ halothane during surgical treatment and $0.5 \%$ halothane during recording. The end-tidal $\mathrm{CO}_{2}$ was continuously monitored with a gas monitor (San-ei, 1H31) and maintained at 3.8$4.3 \%$. The body temperature was controlled at $38 \pm$ $0.5^{\circ} \mathrm{C}$ with a thermostatically controlled heating pad.

The animals were mounted in a stereotaxic frame. Applying the usual clinical procedure, enucleation of the left eyeball and craniotomy were performed on the left side to expose the anterior coronal gyrus, which is the oro-facial area of the SI. After the fixation of a metallic chamber to the cranium with acrylic resin and removal of the dura, the inner cavity of the chamber was filled with warmed paraffin oil to minimize the pulsation. Cortical EEG, EKG, and blood pressure in the femoral artery were continuously monitored as indicators of the physiological state of the animals, and were checked to maintain a sufficient level of anesthesia. Under these conditions, there were no obvious changes in the indications even when the intensity of tooth pulp stimulation was increased to $0.7 \mathrm{~mA}$, indicating sufficient anesthesia of the animals. The conditions of the experiment were in agreement with the ethical guidelines of the International Association for the Study of Pain for investigations of experimental pain.

Test stimulation. In each animal, the bilateral upper and lower canines and molars were prepared $(8$ teeth) for electrical stimulation of the tooth pulp. The procedure for implanting the stimulating electrodes into the teeth was basically the same as that described in our previous paper [9]. Single rectangular pulses, $0.5 \mathrm{~ms}$ in duration, were bipolarly delivered through an isolator (Nihon Kohden, MSE-3R) in a regular sequence to the 8 tooth pulps. The threshold for the jawopening reflex (JOR) was measured under ketamine anesthesia before immobilization. The stimulus intensity was typically maintained at less than $3-5$ times the threshold for the JOR; the maximum values did not exceed $430 \mu \mathrm{A}$. The intensity of the exploratory stimulus to detect TPD neurons was usually between 100 and $300 \mu \mathrm{A}$. When the tooth pulp stimulation excited the neurons, pressure stimulation using a wooden rod was also applied manually to the tooth having afferents to this neuron. Neurons responding to pressure stimulation were excluded from the data analysis due to the possibility of the stimulus current spreading to the periodontium. Tactile stimulation was delivered manually to the facial skin and oral mucosa with a fine paintbrush and glass rod.

Conditioning stimulation. At first, a concentric bipolar electrode (Unique Medical, S-121) for conditioning stimulation was placed in the amygdala (anterior 11.0-13.0, lateral 8.0-12.0, vertical -1.0 to $-5.0)$. Subsequently, the electrode was moved to the location where the maximum JOR inhibition was obtained. The reason for this is that the ACE conditioning stimulation inhibited the JOR induced by tooth pulp stimulation [11]. The stimulation was a train of 33 rectangular pulses $0.2 \mathrm{~ms}$ in duration with a frequency of $330 \mathrm{~Hz}$ at intervals of $8-10 \mathrm{~s}$; the intensity was usually $300 \mu \mathrm{A}$. The conditioning-test (C-T) interval (between the cessation of conditioning and the 
onset of test stimuli) was usually $10-50 \mathrm{~ms}$. The stimulating side was homolateral to the recording site in the SI.

ACE stimulation alone under the same conditions as that of the conditioning stimulation did not exert any influence on blood pressure or heart rate. Additionally, the stimulation did not change the rate of spontaneous discharge of F-type or S-type TPD neurons nor non-nociceptive neurons, except for one Ftype TPD neuron whose discharge rate was increased. In addition, abnormal discharges such as epileptic seizures did not appear on the EEG after ACE stimulation.

Recording. Extracellular single unit recordings in the left SI were made with a glass capillary microelectrode (7-10 M $\Omega$ ) filled with 2\% Pontamine Sky Blue in $1 \mathrm{M}$ sodium acetate. The electrode was advanced in steps of $3-5 \mu \mathrm{m}$ by a digital hydraulic microdrive (Narishige, PC5N). Neuronal activities were fed into an amplifier (Nihon Kohden, VC-11), and these analogue signals were then analyzed by an averager/histogram analyzer (Nihon Kohden, QC-111J) that produced post-stimulus time (PST) histograms of the unit responses to tooth pulp stimulation. Isolation of single units was achieved by visual monitoring of the action potential on an oscilloscope, and PST histograms were generated by means of a window discriminator. The signals were also sent to a loudspeaker for auditory monitoring.

Application of drugs. Two kinds of receptor antagonists were injected into the left femoral vein. The opioid antagonist naloxone (Sigma Chemical Co., USA) was dissolved at $1 \mathrm{mg} / 0.2 \mathrm{ml}$ saline. This drug was administered to 5 cats at a dose of $1 \mathrm{mg} / \mathrm{kg}$. The histamine $\mathrm{H}_{1}$ antagonist diphenhydramine hydrochloride (Kowa Co., Japan) was dissolved at $0.5 \mathrm{mg} / 0.2 \mathrm{ml}$ saline. This drug was given to 5 cats at a dose of $0.5 \mathrm{mg} / \mathrm{kg}$. The vehicle (saline) was administered in an equal volume to each animal. Drugs and saline injections were carried out slowly over a period of about $1 \mathrm{~min}$. At 25, 45 and $65 \mathrm{~min}$ after drug injections, unit responses to the tooth pulp stimulation were measured without ACE conditioning stimulation.

Histological analysis. Recording sites of unit activities in the SI were marked by depositing Pontamine sky blue with a cathodal current $(5 \mu \mathrm{A}, 25 \mathrm{~min})$ applied through the microelectrode. The stimulating sites in the amygdaloid nucleus were marked by depositing iron ions from the concentric stimulating electrodes with an anodal current $(3 \mu \mathrm{A}, 20 \mathrm{~s})$. At the termination of each experiment, animals were sacrificed with an overdose of pentobarbital and then perfused transcardially with normal saline solution fol- lowed by $10 \%$ formalin containing $2 \%$ potassium ferrocyanide. Prussian blue reactions were induced by the iron ions in the amygdala. The brain was removed and stored for at least $48 \mathrm{~h}$ in $10 \%$ formalin containing $20 \%$ sucrose. Frozen coronal sections of $50 \mu \mathrm{m}$ in thickness were taken from the SI and amygdala. They were mounted on gelatin-coated slides and then stained with cresyl violet. The recording sites in the SI and the stimulating sites in the amygdala were determined by histological examination of the serial sections. Sites of stimulation were plotted onto a series of camera lucida drawings of the amygdala. The nomenclature and main divisions of the amygdala were adopted from the descriptions of Jasper and AjimoneMarsan [12] and Krettek and Price [13].

Data analysis. The total number of impulses evoked by tooth pulp stimulation was counted before (control) and after (recovery) ACE conditioning stimulation. The mean response to tooth pulp stimulation after ACE conditioning stimulation was expressed as a percentage of the control response. The TPD neurons that yielded more than a $20 \%$ reduction in the response to tooth pulp stimulation after ACE conditioning stimulation are referred to as inhibited-TPD neurons.

In antagonist-injection experiments, the response of TPD neurons to tooth pulp stimulation before application of a drug was taken as the control response. Tooth pulp stimuli with and without ACE conditioning stimulation were applied alternatively, every $5 \mathrm{~min}$, after application of the drugs. The relative firing responses to the pulpal stimuli were given as a percentage of the control response. All quantitative results are expressed as mean \pm SD. Statistical comparisons were made using Student's $t$-test. Values of $p<0.05$ were considered statistically significant.

\section{RESULTS}

\section{Tooth pulp-driven (TPD) neurons in the first somatosensory cortex (SI)}

Seventy-nine TPD neurons were recorded from the oro-facial area of the SI located in the anterior coronal gyrus. Figure 1 shows a histogram illustrating the latency of the firing response to tooth pulp stimulation. The TPD neurons were clearly divided into two clusters, F-type (black column) and S-type (hatched column), by their latencies of less or more than $20 \mathrm{~ms}$. Of 79 TPD neurons, 18 were F-type TPD neurons with short latencies of between 8 and $20 \mathrm{~ms}(10.8 \pm 4.4 \mathrm{~ms}$, $n=18$ ), and 61 were S-type TPD neurons with long latencies of between 22 and $108 \mathrm{~ms}(40.7 \pm 16.0 \mathrm{~ms}, n=$ $61)$. 


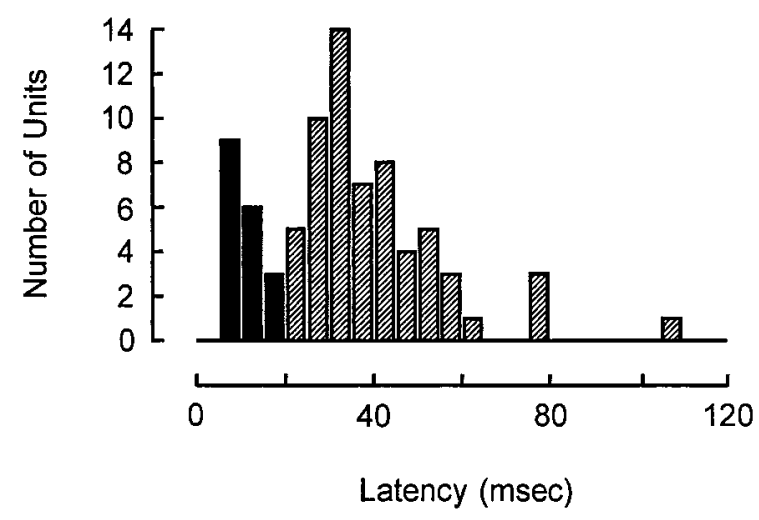

Fig. 1. Distribution of latencies for firing responses to electrical stimulation of tooth pulp in TPD neurons. Black and hatched columns indicate F-type and S-type TPD neurons, respectively.

None of the TPD neurons recorded in the present study exhibited any afterdischarge following tooth pulp stimulation, although we previously reported $\mathrm{Fa}$ type TPD neurons accompanied by afterdischarges [9]. An increase in stimulus intensity to the tooth pulp did not change the temporal firing patterns in the TPD neurons. In addition, all of the TPD neurons were spontaneously active with a firing rate of less than 9.4/s.

About $20 \%$ of the TPD neurons received lowthreshold mechanoreceptive and pulpal (nociceptive) inputs, indicating that these TPD neurons have a wide dynamic range. Cutaneous receptive fields of those TPD neurons were confined to the hairy or glabrous skin of the lip, mucous membrane of the oral cavity, or periodontal membrane(s). Of the 18 F-type TPD neurons, 10 were tooth pulp-specific and 4 had other cutaneous receptive fields. The remaining 4 neurons were not tested as to whether they had a cutaneous receptive field. Although one F-type neuron received 6 pulpal inputs, the majority of F-type neurons received pulpal input(s) from one to three teeth $(1.8 \pm 1.5$ teeth, $n=10$ ). The F-type TPD neurons received a more dominant projection from the contralateral teeth than from the ipsilateral ones. Of the 61 S-type TPD neurons, 31 were tooth pulp-specific and 10 received cutaneous inputs. The remaining 20 neurons were not tested for a cutaneous receptive field. These neurons had input connections with $1-8$ teeth $(3.6 \pm 2.3$ teeth, $n=35$ ).

\section{Effects of ACE conditioning stimulation on S- type TPD neurons}

The conditioning stimulation in the area outside the ACE did not have any significant influence on the patterns of firing of S-type TPD neurons in response to pulpal stimulation $(n=42)$.

In 35 out of 61 S-type neurons, ACE conditioning stimulation significantly reduced the frequency of firing in response to the test pulpal stimulation. Of the inhibited S-type neurons, 17 were tooth pulp-specific and 4 received cutaneous inputs. The remaining 26 neurons showed no effect. The conditioning stimulation was applied at varying $\mathrm{C}-\mathrm{T}$ intervals. The maximal inhibition was obtained when a conditioning stimulation was applied $10-50 \mathrm{~ms}$ prior to the test stimulus. The inhibitory effect diminished gradually and was abolished at a C-T interval of 500-2,000 ms. Thus, conditioned responses were obtained at a C-T interval of $10-50 \mathrm{~ms}$ in all subsequent experiments.

Figure 2A shows a typical example of the effects of ACE conditioning stimulation on the firing rate of an S-type neuron (latency for the firing response, $42 \mathrm{~ms}$ ). Each PST histogram represents an accumulated response of 20 trials. At C-T intervals of $10 \mathrm{~ms}$, the response to tooth pulp stimulation with a peak time of $60 \mathrm{~ms}$ was almost completely abolished. This neuron was sensitive only to pulpal stimulation. Another neuron was obtained from the same animal. The neuron responded to tactile stimulation of the upper lip by a paintbrush but did not respond to the noxious pinch of the area by serrated forceps. In this neuron, the responses to electrical stimulation of the upper lip were not affected by conditioning stimulation of the same ACE site (Fig. 2B). The site of the conditioning stimulation was in the most-ventromedial part of the ACE (Fig. 2C). The effects of ACE conditioning stimulation on the response to non-noxious stimulation were examined in 12 neurons in the same manner. None of the neurons were influenced by the conditioning stimulation.

Some of the S-type neurons received input(s) from the bilateral tooth (or teeth). ACE conditioning stimulation similarly inhibited the firing response to the stimulation of the ipsilateral as well as the contralateral tooth pulp. The inhibitory effects on the contralateral and ipsilateral teeth were $72.8 \pm 24.3 \%(n=22)$ and $77.9 \pm 20.7 \%(n=11)$, respectively, and there was no significant difference between them.

Figure 3 shows the latencies for firing responses to pulpal stimuli in inhibited and non-inhibited S-type neurons following ACE conditioning stimulation. The non-inhibited group (lower part of Fig. 3) tended to have shorter latencies compared with the inhibited group (upper part of Fig. 3). The mean latencies of 26 non-inhibited S-type neurons and 35 inhibited ones were $34.8 \pm 10.5$ and $45.1 \pm 18.0 \mathrm{~ms}$, respectively. There was a statistically significant difference (nonpaired $t$-test, $p<0.01$ ) even though the one neuron 
A $\quad$ B

IPSI. UPPER CANINE
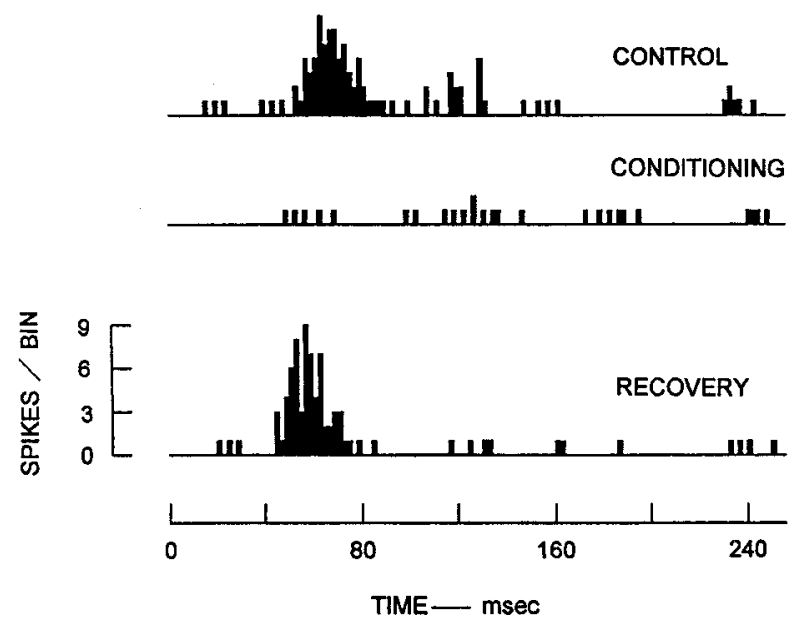

CONTRA. UPPER LIP
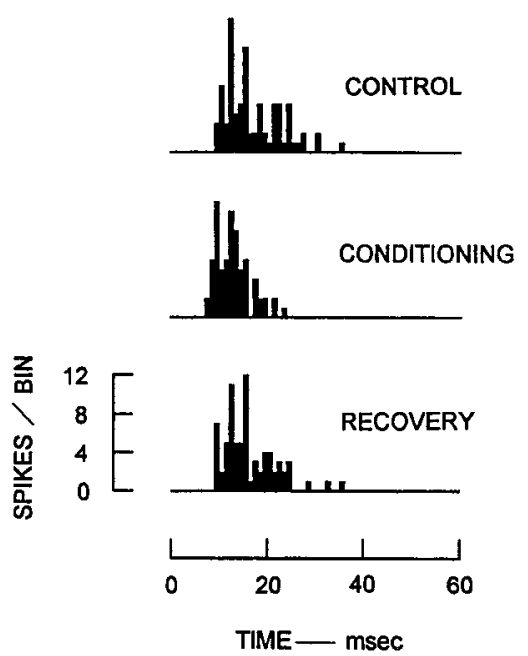

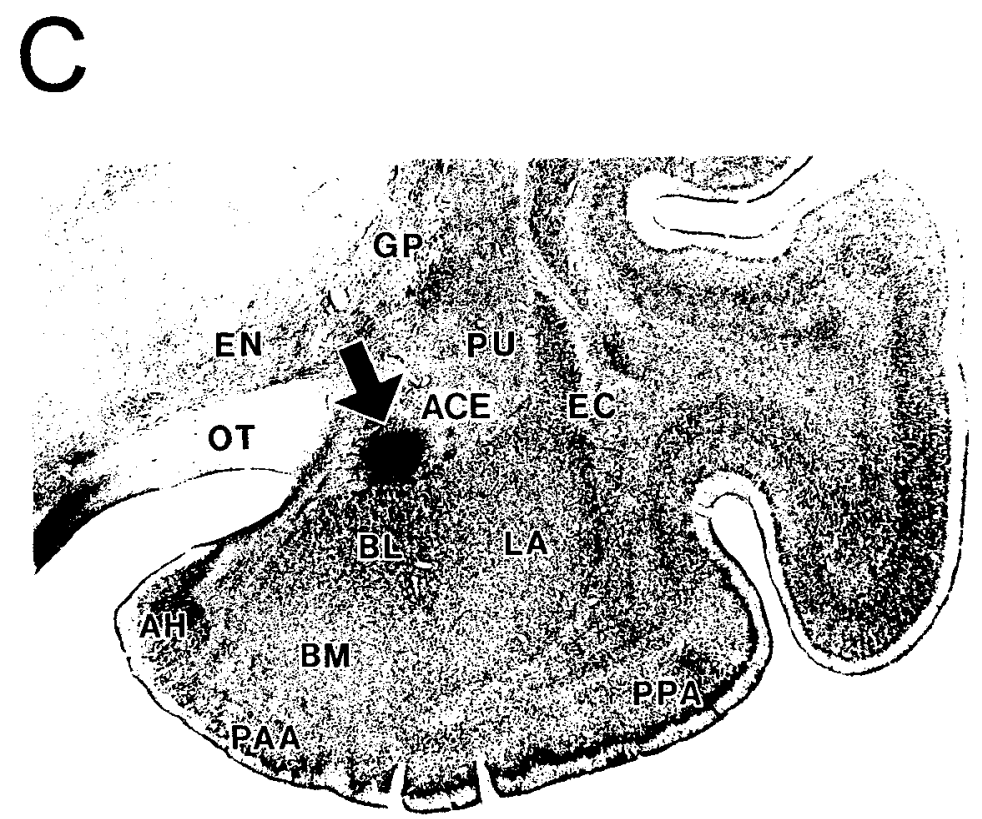

Fig. 2. Effects of ACE conditioning stimulation on TPD neurons. A: Inhibitory effect of ACE conditioning stimulation $(300 \mu \mathrm{A})$ on the firing response of an S-type neuron to tooth pulp stimulation (ipsilateral upper canine, $200 \mu \mathrm{A}$ ). This neuron was tooth pulp-specific. The response was inhibited at a conditioning-test interval of $10 \mathrm{~ms}$ (middle trace). In the PST histogram, the ordinate shows the number of spikes/bin and the abscissa shows the time course in milliseconds (bin width: $2 \mathrm{~ms}$ ). Twenty trials were accumulated for each histogram. B: Effect of ACE conditioning stimulation on a nonnociceptive (tactile) neuron. Electrical stimulation was ap- plied to the contralateral upper lip as a non-noxious stimulus (bin width: $1 \mathrm{~ms}$ ). The non-nociceptive response was not affected by ACE stimulation at the same position as that shown in Fig. 1A. C: Location of the conditioning stimulation in Fig. $1 \mathrm{~A}$ and $\mathrm{B}$. The stimulation site was in the most-ventromedial part of the ACE (arrow). AH, amygdalo-hippocampal area; BL, basolateral amygdaloid nucleus; BM, basomedial amygdaloid nucleus; EC, external capsule; EN, entopeduncular nucleus; GP, globus pallidus; LA, lateral amygdaloid nucleus; OT, optic tract; PAA, periamygdaloid area; PPA, prepiriform area; PU, putamen.

two different S-type TPD neurons with different firing patterns. The S-type neuron on the left responded with a peak time of approximately $60-65 \mathrm{~ms}$, and response with the longest latency $(108 \mathrm{~ms})$ in the inhibited group was excluded. Figure 4 shows the effect of conditioning stimulation of the same site in the ACE on 


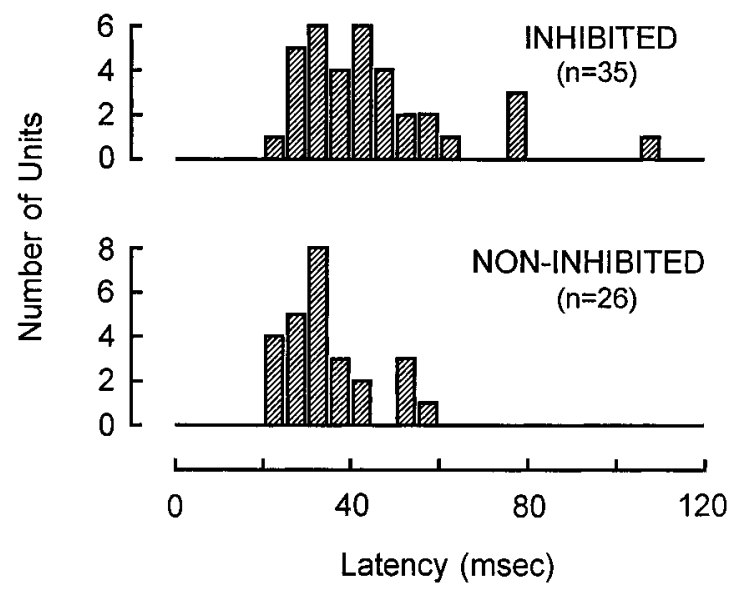

Fig. 3. Comparison of latencies for firing responses to pulpal stimulation in inhibited (upper) and non-inhibited (lower) S-type TPD neurons by ACE conditioning stimulation.

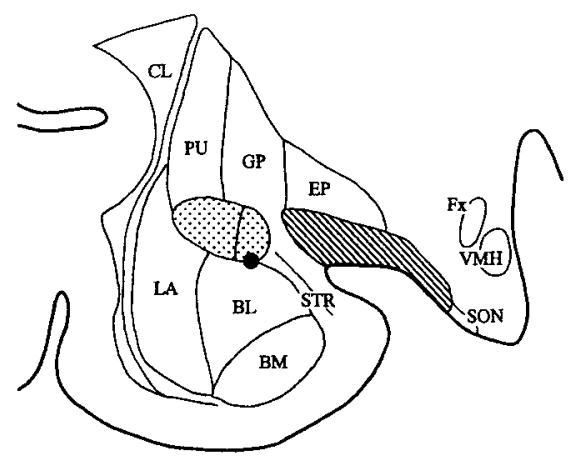

IPSI. UPPER MOLAR

CONTROL

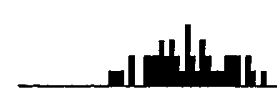

CONDITIONING

alnew

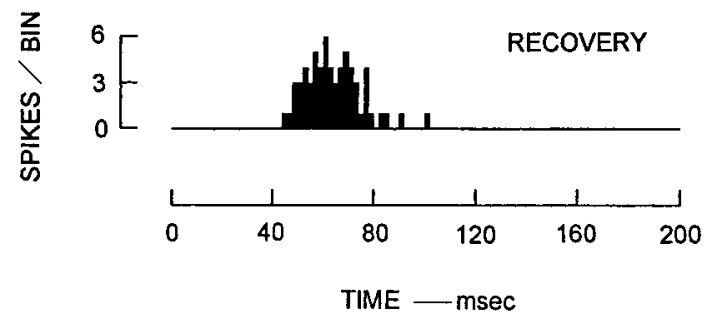

Fig. 4. Effects of ACE conditioning stimulation on two S-type TPD neurons. Firing activities of the S-type neuron with a long peak time (left) but not the S-type neuron with short peak time (right) were inhibited by conditioning stimulation of the same site in the ACE (closed circle in top trace). Ipsilateral upper molar $(200 \mu \mathrm{A})$ and ipsilateral lower canine was inhibited to $43 \%$ of the control response by ACE conditioning stimulation. In contrast, the S-type neuron on the right showed firing with a peak time of 40 $42 \mathrm{~ms}$, and the same ACE conditioning stimulation did not markedly alter the firing rate.

\section{Effects of ACE conditioning stimulation on F- type TPD neurons}

Next, we examined the effect of ACE conditioning stimulation on the firing response in F-type neurons in terms of contralateral or ipsilateral stimulation. The conditioning stimulation of the ACE and of other areas did not exert any significant influence on the frequency of firing induced by the pulpal stimulation in 17 out of 18 F-type neurons. Only one remaining neuron, with an $18 \mathrm{~ms}$ latency, was inhibited to $93.5 \%$ of
IPSI. LOWER CANINE
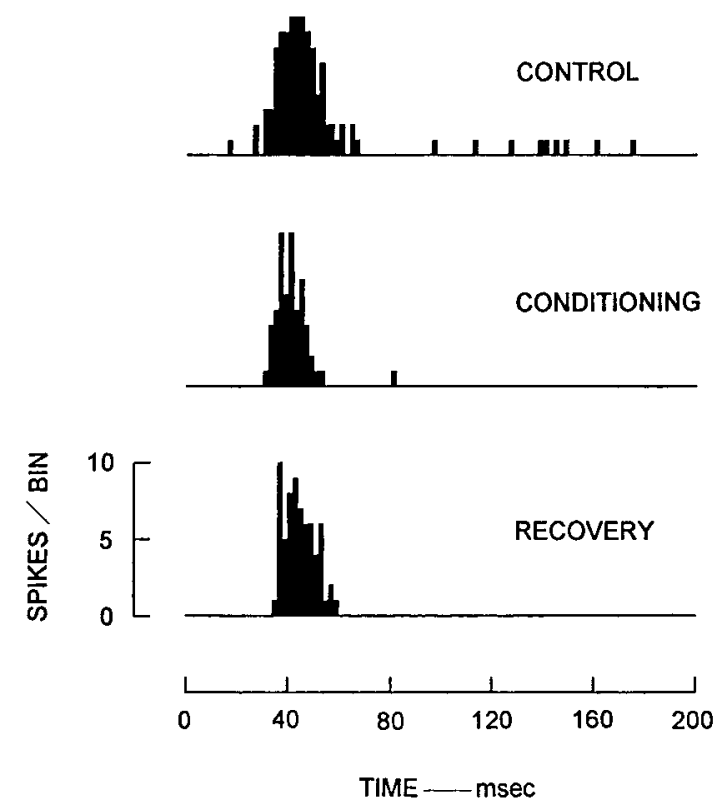

$(400 \mu \mathrm{A})$ were stimulated right and left, respectively. Twenty trials were accumulated for each histogram (bin width: $2 \mathrm{~ms}$ ). CL, claustrum; EP, entopeduncular nucleus; Fx, fornix; SON, supraoptic nucleus; STR, stria terminalis; VMH, ventromedial hypothalamic nucleus; the other abbreviations are the same as those listed in Fig. 2. 


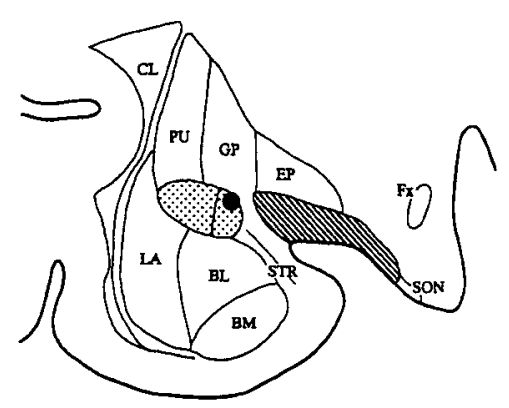

Slow-type
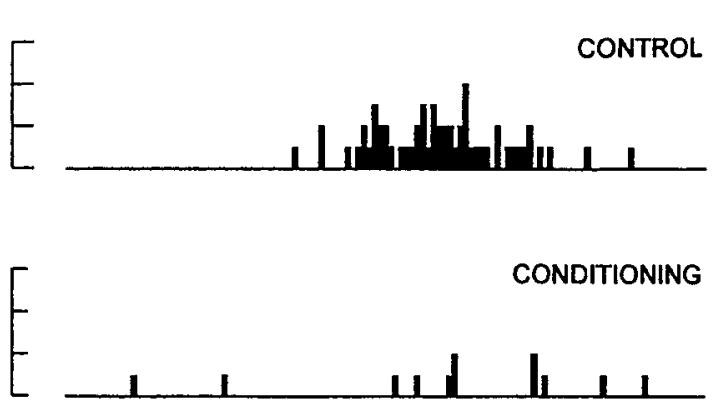

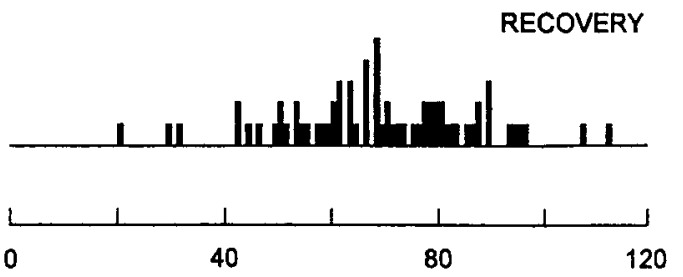

TIME - msec

Fig. 5. Effect of ACE conditioning stimulation on Stype and F-type TPD neurons. Firing activities of the S-type neuron (left) but not of the F-type (right) were inhibited by ACE stimulation (closed circle in the top trace). Ipsilateral lower molar $(200 \mu \mathrm{A})$ and ipsilateral upper canine $(50 \mu \mathrm{A})$

the control.

In three experiments, we recorded the firing rates of both S-type and F-type neurons in the same animal, and the effects of conditioning stimulation of the same site in the ACE were investigated. In all cases, ACE conditioning stimulation reduced the firing rate of $\mathrm{S}$ type neurons $(n=3)$ but not that of F-type neurons $(n=4)$. One typical sample is presented in Fig. 5. The S-type neuron with a latency of $42 \mathrm{~ms}$ was inhibited to $18.3 \%$ of the control response by ACE conditioning stimulation (left part of Fig. 5), whereas the F-type neuron with a latency of $8.0 \mathrm{~ms}$ was not altered at all by stimulation (right part of Fig. 5).

\section{Fast-type}
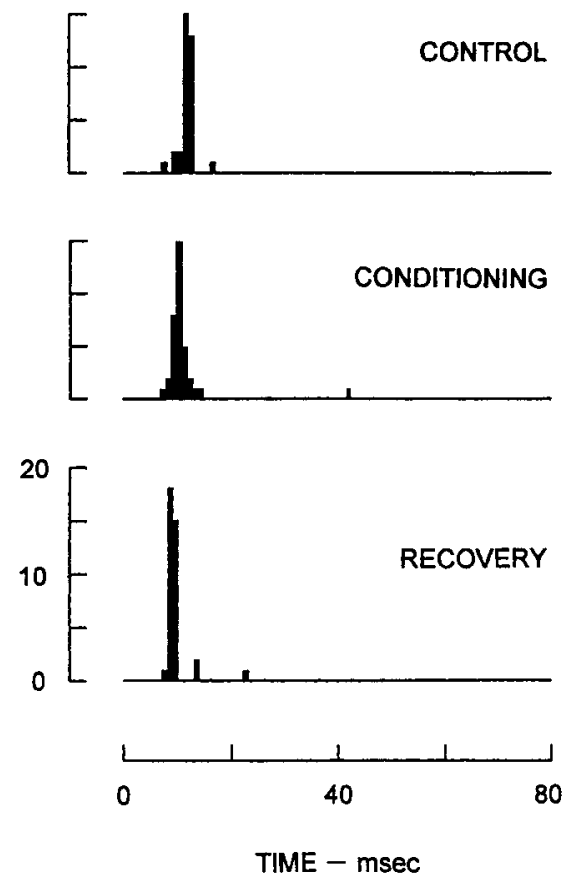

were stimulated for S-type and F-type neurons, respectively. Twenty trials were accumulated for each histogram (bin width: $2 \mathrm{~ms}$ ). Abbreviations are the same as those listed in Figs. 2 and 4.

\section{Differential effects of ACE conditioning stimu- lation on S-type and F-type neurons}

Figure 6 shows the distribution of relative firing rates after ACE conditioning stimulation against the latency for firing response to tooth pulp stimulation in 18 F-type and 58 S-type neurons. None of the F-type TPD neurons, except one with a latency of $18 \mathrm{~ms}$, were significantly affected by ACE conditioning stimulation; that is, firing responses of F-type neurons to tooth pulp stimulation were not attenuated by ACE stimulation to $<80 \%$ of the control. In contrast, responses of S-type neurons were inhibited by ACE stimulation to $76-0 \%$ of the control $(n=32)$, and 20 out of the 32 inhibited TPD neurons were inhibited to $<30 \%$ of the control. The mean maximal inhibition in 32 S-type neurons was $73.9 \pm 23.1 \%$. There was no 


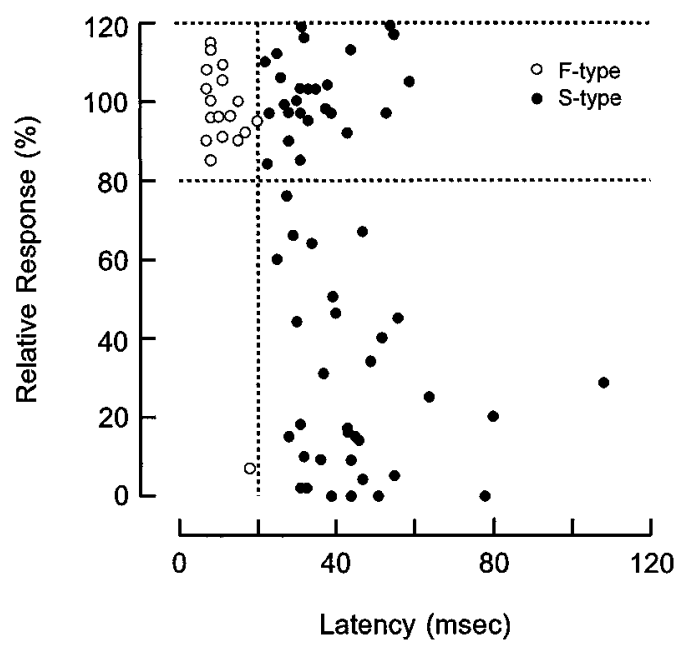

Fig. 6. Scatter diagram plotting of latencies for firing responses (ms) against relative inhibitions by ACE conditioning stimulation in 18 F-type and 58 S-type TPD neurons. The inhibitory effect produced by ACE conditioning stimulation $(300 \mu \mathrm{A})$ is shown as the relative response to that of the control (\%). The dashed vertical line represents a line of $20 \mathrm{~ms}$ in latency dividing F-type and S-type neurons. The dashed horizontal lines ( $\pm 20 \%$ of control) represent the points at which the firing activity of TPD neurons is significantly increased $(>120 \%)$ or inhibited $(<80 \%)$ by ACE conditioning stimulation.

significant correlation $(r=0.121 ; 0.05<p<0.10)$, however, between the inhibition of firing rate produced by ACE stimulation and the latency in 33 inhibited neurons (1 F-type and 32 S-type).

\section{Actions of naloxone and diphenhydramine hydrochloride on ACE inhibitory effects}

The involvement of opioid receptor activation in the inhibitory effect of ACE conditioning stimulation was investigated in 5 S-type neurons of 5 cats. Intravenous injection of naloxone $(1 \mathrm{mg} / 0.2 \mathrm{ml}$ saline $/ \mathrm{kg})$, a nonselective opioid receptor antagonist, did not affect the inhibitory effect of ACE stimulation on the S-type neuron for a period of $60 \mathrm{~min}$ (top trace in Fig. 7). In addition, naloxone alone did not significantly influence unit response to tooth pulp (test) stimulation without ACE conditioning stimulation.

To evaluate whether histamine $(\mathrm{H})$ receptors play a role in the ACE inhibitory effect, diphenhydramine hydrochloride, an $\mathrm{H}_{1}$-receptor antagonist, was injected intravenously $(0.5 \mathrm{mg} / 0.2 \mathrm{ml}$ saline $/ \mathrm{kg})$. This drug partially antagonized the ACE inhibitory effect in all 5 S-type TPD neurons tested (bottom trace in Fig. 7). The inhibition of unit responses by ACE conditioning stimulation was $19.1 \pm 4.2 \%$ of the control before and $56.0 \pm 15.1 \%$ of the control $20 \mathrm{~min}$ after administration of the antagonist; $46 \%$ of the ACE inhibitory ef-

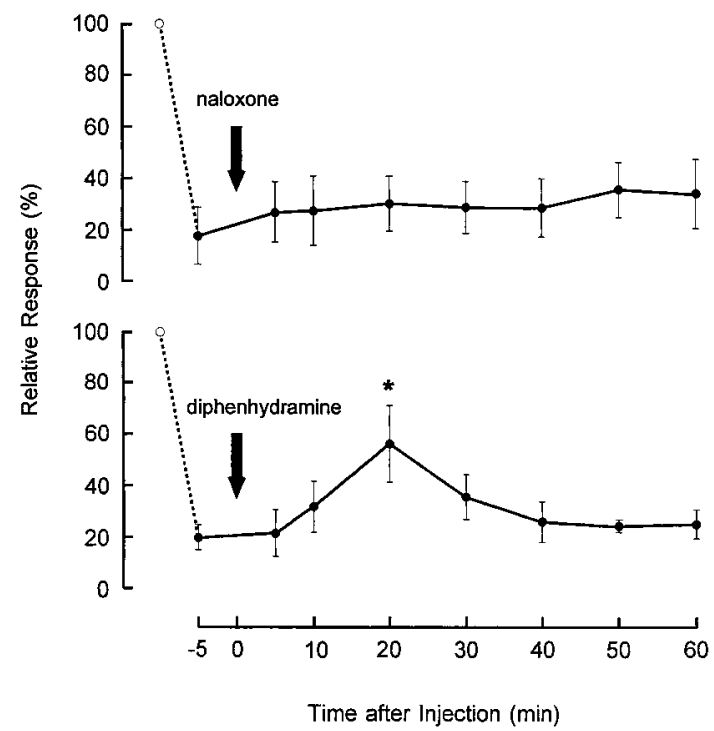

Fig. 7. Effect of naloxone and diphenhydramine hydrochloride on the inhibition induced by ACE conditioning stimulation. The ACE inhibitory effect was not influenced by the opioid antagonist ( $1 \mathrm{mg} / \mathrm{kg}$, I.V., $n=5$, top trace), whereas the inhibitory effect was partially antagonized by the histamine $\mathrm{H}_{1}$ antagonist $(0.5 \mathrm{mg} / \mathrm{kg}$, I.V., $n=5$, bottom trace). The ordinate shows the mean percent changes in the number of spikes with ACE conditioning stimulation, and the abscissa shows the time in minutes after drug injection. The vertical bar at each point indicates the SEM. * Significantly different from the pre-injection value at $p<0.05$, $t$-test.

fect was antagonized by the drug. The differences between these values were statistically significant $(p<$ $0.05)$. The antagonistic effect disappeared at $40 \mathrm{~min}$ after administration. The drug did not significantly influence firing response to tooth pulp (test) stimulation without ACE conditioning stimulation. Saline injection in the same manner produced only a negligible effect. Finally, a histological analysis revealed that the tip of the electrode for conditioning stimulation was located in the medial part of the ACE in all animals examined.

\section{Distribution of conditioning stimulation sites}

If the conditioning electrode was moved over $1.5 \mathrm{~mm}$ dorsally or ventrally, an inhibitory effect was not observed. This finding indicates that the effective area for producing inhibition may be relatively confined. Figure 8 shows the distribution of 54 stimulating sites that were histologically reconstructed on the basis of Prussian blue reactions. The tip positions of 27 stimulating electrodes were completely or partially within the medial division of the ACE, and those of 12 electrodes were in the lateral division of the ACE. The remaining 15 electrodes were located in areas other than the ACE: 5 in other amygdaloid nuclei and 

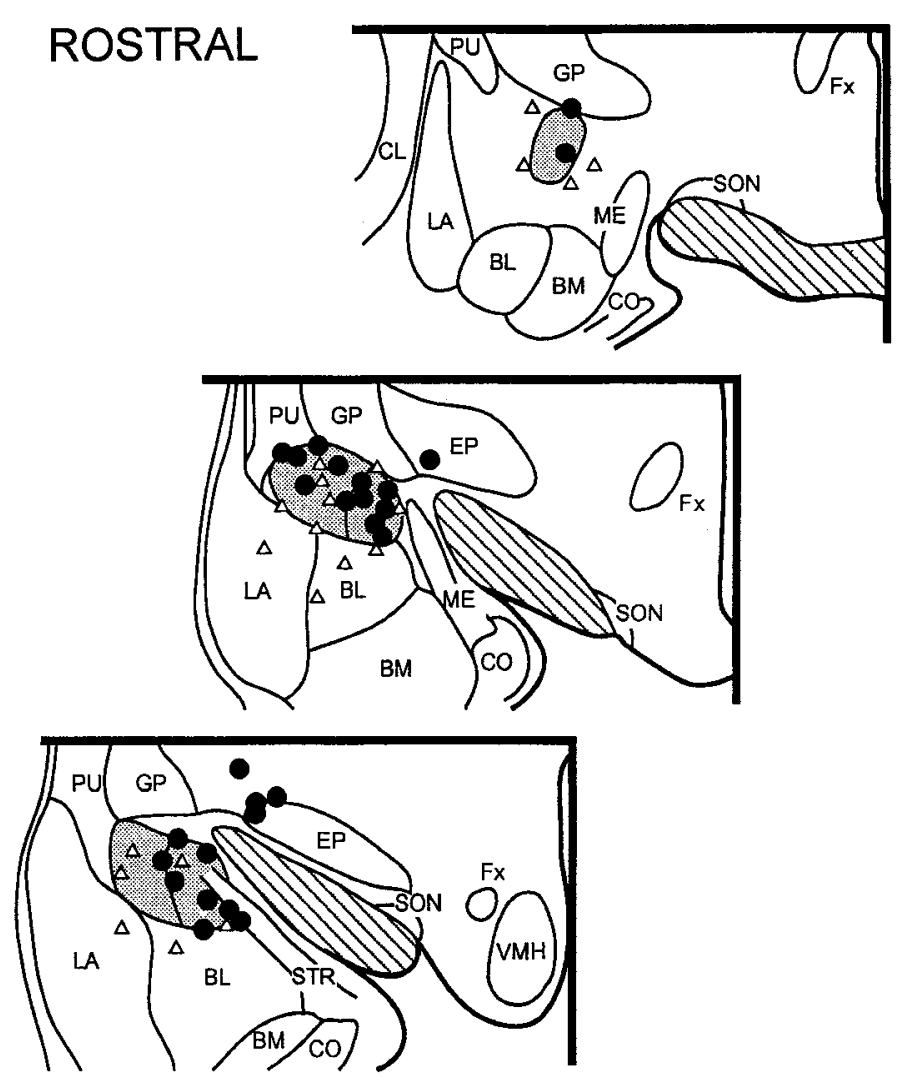
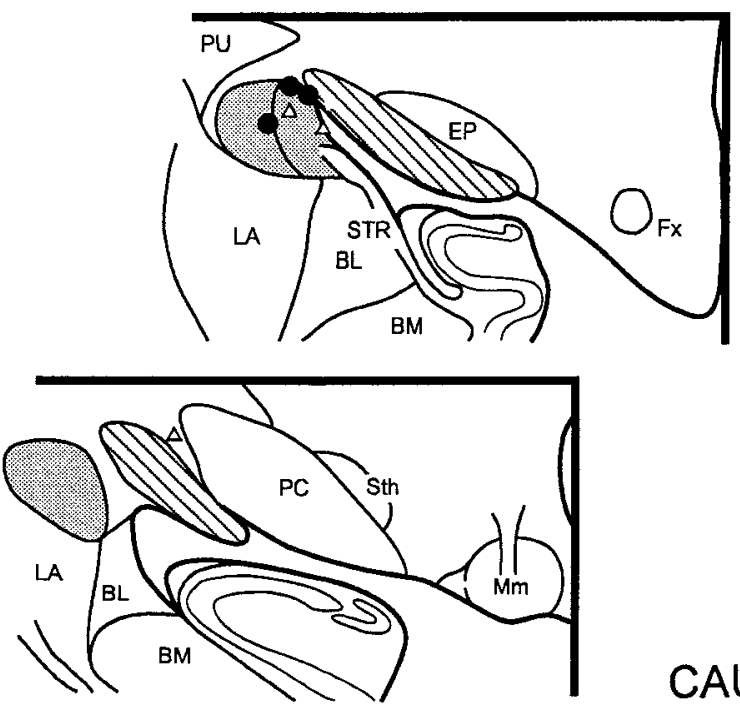

10 in areas other than amygdaloid nuclei. Moreover, effective sites were concentrated in the medial part of the ACE. Thus, an inhibitory effect on the firing activities of TPD neurons (including one F-type neuron) was observed when the stimulating electrodes were located in 20 out of 27 positions $(74.1 \%)$ in the medial part and 5 out of 12 positions (41.7\%) in the lateral part of the ACE. Conditioning stimulation of other amygdaloid nuclei (lateral, basomedial and basolateral nuclei) did not exert any influence on firing activity.
Fig. 8. Histological locations of the tips of 54 stimulating electrodes in coronal sections. Filled circles indicate stimulating sites that produced inhibition of the nociceptive response of TPD neurons (all S-type and all but one F-type neuron). Ineffective stimulating sites are indicated by open triangles. Gray areas and hatched areas show the ACE and optic tract, respectively. Diagrams were adapted from the atlas of Jasper and AjimoneMarsan [12]. CO, cortical amygdaloid nucleus; Mm, mammillary body; Me, medial amygdaloid nucleus; PC, cerebral peduncle; Sth, subthalamic nucleus; The other abbreviations are the same as those listed in Figs. 2, 4 and 5.

\section{DISCUSSION}

Tooth pulp stimulation and TPD neurons in the SI. The nerve endings in teeth can provide nonnociceptive information such as tooth contact in the cat [14], and electrical stimulation of the tooth pulp induces sensations other than pain (such as tingling, warm and cold sensations) in humans [15]. On the other hand, it has been reported that the threshold intensity for escape elicited by tooth pulp stimulation is about 3 times the threshold intensity for the JOR in conscious cats [16]. In addition, the appearance of the 
JOR is coincident with the activation of A $\delta$ fibers in the cat tooth pulp [17], and the appearance of digastric activity is also associated with A $\delta$ activation in the infraorbital nerves [18]. Moreover, tooth pulp stimulation with an intensity identical to that used in this study induces $c$-fos expression in the marginal layer of the subnucleus caudalis, which has been shown to contain nociceptive-specific neurons [19]. It is therefore likely that the firing responses of cortical TPD neurons to tooth pulp stimulation in this study are involved in nociception.

ACE and antinociception. The antinociceptive effect of ACE stimulation observed in this study is strikingly different from analgesia following electroconvulsive shock that produces amygdaloid kindling. Analgesia induced by shock lasted approximately 10-15 min after stimulation and was reversed with systemic naloxone injection [20,21]. In contrast, the ACE inhibitory effect was not reversed by naloxone, and the duration of inhibition was probably in the order of milliseconds. Furthermore, ACE conditioning stimulation did not induce any abnormal discharge such as epileptic seizure as observed in the EEG measurement.

ACE conditioning stimulation produced inhibition of the firing activities in S-type TPD neurons. The present findings suggest that the excitation of ACE neurons induces antinociception, though this has not been confirmed by experiments using an excitatory amino acid. Behavioral studies suggested that the $\mathrm{ACE}$ in rats is involved in nociception. Thus, the microinjection of neurotensin or an enkephalinase inhibitor into the ACE produces an antinociceptive effect $[7,8]$ in the hot-plate test. In addition, the microinjection of cholinergic agonists into the ACE induces an antinociceptive effect in the tail-flick test, although this effect is weak compared with that produced by injection into the medial nucleus of the amygdala [22]. The antinociception produced by exposure to footshock is attenuated by bilateral ACE lesions [23]. The ACE has been demonstrated to be critical for the expression of conditioned antinociceptive responses in both tail-flick and formalin tests [24, 25]. These behavioral results are comparable to the present findings of ACE stimulation exerting an inhibitory influence on nociception. On the other hand, ACE stimulation produced by drugs did not evoke any hyperalgesia in these behavioral studies [24, 25]. Similarly, ACE conditioning stimulation did not produce any facilitation of firing activities in either S- or F-type neurons.

In cats, the ACE has been classified into medial and lateral subdivisions by Nissl and Golgi staining [13,
26]. The present results demonstrate that effective sites in the ACE for antinociception were concentrated mainly within the medial subdivision. Morphological studies have demonstrated that amygdaloid descending projections originate predominantly in the medial subdivision of the ACE. For example, brainstem regions such as midbrain periaqueductal gray (PAG) [27], solitary tract nucleus [28], and parabrachial nucleus [13] receive projections from the medial subdivision of the ACE. An autoradiographic study has indicated that the ACE has direct efferent connections via the ventral amygdalofugal fibers with the dorsal raphe nucleus, nucleus raphe magnus, and cuneiform nucleus in addition to these nuclei [29]. All these nuclei have been concerned in the descending and/or ascending control of nociceptive transmission at the spinal cord or thalamus levels (see Fields and Besson [30] for review).

\section{ACE inhibitory effects on S-type TPD neu-}

rons. The electrical conditioning stimulation of ACE suppressed the firing responses to tooth pulp stimulation in S-type TPD neurons but not those in Ftype neurons. These conditioning effects on TPD neurons are similar to the action of morphine. Morphine markedly suppresses the firing responses to tooth pulp stimulation in S-type neurons with no effect on F-type neurons [31]. The different effects of morphine and ACE conditioning stimulation on S-type and F-type TPD neurons strongly suggest that nociceptive information from the tooth pulp can reach the SI via at least two distinct pathways. Furthermore, the S-type neuron has the firing properties of a long latency, a large number of pulpal afferents, and a stimulus-response function with a steeper slope compared with those of the F-type neuron [9]. Taken together, it appears likely that F-type neurons receive pulpal information through the nucleus ventralis posteromedialis (VPM), which receives direct projections exclusively from the trigeminal sensory nuclear complex, whilst S-type neurons receive projections from medial intralaminar thalamic (MIT) nuclei that receive indirect pulpal afferents from the brainstem reticular formation. There are TPD neurons and nociceptive neurons receiving information from the facial area in the VPM and MIT nuclei [32-35]. TPD neurons in the nucleus centralis lateralis of the MIT receive projections from the nucleus reticularis ventralis of the caudal medulla oblongata [33].

In the present study, approximately $40 \%$ of the Stype neurons tested were not inhibited by ACE conditioning stimulation. The non-inhibited S-type neurons tended to have shorter latencies than those of the inhibited group. The reason why some of the S-type 
neurons were not influenced by ACE conditioning stimulation is unclear. There is a strong possibility that the S-type neurons with relatively short latencies receive input via the VPM rather than the MIT. Indeed, Woda et al. [32] observed that the VPM contains two groups of TPD neurons: one group with a latency of less than $12 \mathrm{~ms}$ and the other having a latency of 12 to $46 \mathrm{~ms}$.

We could not identify the site where the stimulation of ACE inhibits nociceptive transmission. Fanselow and Kim [2] suggested that a part of conditioned fearinduced analgesia depends on blocking ascending pain information in the dorsal horn of the spinal cord. In addition, Kapp et al. [36] suggested that the ACE influenced neocortical arousal by modulating the activity of cholinergic neurons in the nucleus basalis. The selective inhibition of S-type neuron activity by ACE conditioning stimulation suggests that the modulating site is not in the medullary dorsal horn and SI. If the ACE exerts an inhibitory action on nociceptive neurons in the medullary dorsal horn and SI, it is highly probable that such selectivity for S-type neurons does not exist. It is possible that ACE stimulation-produced inhibition of TPD neurons occurs at the level of the thalamus, and that pulpal (nociceptive) information via the MIT is more sensitive to ACE stimulation. This is consistent with the report that the pain modulatory system to the MIT and nucleus ventralis posterolateralis (VPL) have different characteristics [37]. Further, it seems unlikely that the parabrachial nucleus (PB) receiving ACE inputs is responsible for the inhibitory effect [29]. Stimulation of the PB inhibits cutaneous nociceptive and nonnociceptive responses and spontaneous discharges in the trigeminal subnucleus caudalis [38], whereas ACE stimulation inhibits only nociceptive response. Moreover, our result showing that the ACE inhibitory effect was not changed by naloxone indicated that the descending pain control system from the brainstem is not responsible for the inhibitory effect because opioid participates at the medullary dorsal horn in this system. This speculation is also supported by the behavioral observation that the injection of an enkephalinase inhibitor into the ACE does not affect the tail-flick response mediated by the spinal substrate but affects the hotplate response mediated by the supraspinal substrate [8].

\section{ACE antinociception and stress-induced} analgesia (SIA). Electrical or chemical stimulation of the ACE mimics stress-like responses such as increases in arterial blood pressure, heart rate and respiratory rate $[39,40]$, and it induces ulcer formation [41]. Furthermore, lesions of the ACE attenuate or abolish the autonomic or behavioral changes produced by a variety of stresses $[4,5]$. The administration of CRF seems to lead to a number of responses to stressinduced c-fos expression in the ACE [6]. Thus, ACE seems to play a central role in the control of a variety of stress responses. In addition, footshock stress leads to elevation of the pain threshold in the rat [42]. This phenomenon has been referred to as SIA. The ACE may be one of the candidate sites for producing SIA. The inhibitory effect of ACE conditioning stimulation on the firing response to pulpal stimulation seems to be a neurophysiological basis for SIA.

A number of studies have confirmed the existence of opioid and non-opioid systems of SIA (see Amit and Galina [1] for review). The non-opioid types of SIA are insensitive to naloxone and naltrexone and do not show cross-tolerance to morphine [1]. The present study showed that naloxone did not reverse the inhibitory effect of ACE stimulation; the opioid receptor is not involved in the ACE inhibitory effect. On the other hand, a $\mathrm{H}_{1}$-antagonist, diphenhydramine, partially reversed the ACE inhibitory effect. Lewis et al. [43] observed that the non-opioid type of SIA is antagonized by diphenhydramine in the rat. Additionally, the analgesic effect of an intraventricular injection of histamine is inhibited by an $\mathrm{H}_{1}$-receptor antagonist but not a $\mathrm{H}_{2}$-antagonist in the rat [44]. These findings suggest that $\mathrm{H}_{1}$-receptors are significantly involved in nociception. In the present study, the maximal effect of the $\mathrm{H}_{1}$-antagonist was observed at 20 min after intravenous injection. This long latency suggests that $\mathrm{H}_{1}$-receptors are related indirectly rather than directly to the ACE inhibitory system.

There are other possibilities regarding the involvement of the ACE in SIA. In the opioid type of SIA, opioid receptors may be present in the regions that relay stress information to the ACE. This possibility is supported by the fact that the microinjection of morphine into the basolateral amygdaloid nucleus projecting to the ACE, but not into the ACE itself, induces analgesia in behavioral studies [45]. Manning and Mayer [46] reported that a bilateral lesion of the ACE abolishes morphine-induced antinociception in formalin and tail-flick tests. These findings support the idea that ACE contributes to produce antinociception through opioid receptors. There are reports, however, indicating the involvement of $\mathrm{H}_{2}$-receptors in SIA [47]. Further experiments are needed to confirm whether the ACE is implicated in opioid or non-opioid types of SIA.

We are most grateful to Prof. Y. Kitada for his critical reading of the manuscript. We would also like to thank Dr. P. 
Langman for his comments on the manuscript and his kind assistance concerning English usage. This research was supported by Grants-in-Aid for Scientific Research (Nos. 03670871 and 03771317 ) from the Ministry of Education, Science, Sports and Culture of Japan and by a grant (H854) from the Iwate Medical University Keiryokai Research Foundation.

\section{REFERENCES}

1. Amit $Z$ and Galina ZH: Stress-induced analgesia: adaptive pain suppression. Physiol Rev 66: 10911120, 1986

2. Fanselow MS and Kim JJ: The benzodiazepine inverse agonist DMCM as an unconditional stimulus for fear-induced analgesia: implications for the role of $\mathrm{GABA}_{A}$ receptors in fear-related behavior. Behav Neurosci 106: 336-344, 1992

3. Glavin GB, Murison R, Ovenmier JB, Pare WP, Bakke HK, Henke PG, and Hernandez DE: The neurobiology of stress ulcers. Brain Res Rev 16: 301-343, 1991

4. Hitchcock $J$ and Davis M: Lesions of the amygdala, but not of the cerebellum or red nucleus, block conditioned fear as measured with the potential startle paradigm. Behav Neurosci 100: 11-22, 1986

5. Zhang JX, Harper RM, and Ni H: Cryogenic blockade of the central nucleus of the amygdala attenuates aversively conditioned blood pressure and respiratory responses. Brain Res 386: 136-145, 1986

6. Arnold FJL, De Lucas Bueno M, Shiers H, Hancock DC, Evan GI, and Herbert J: Expression of $c$-fos in regions of the basal limbic forebrain following intracerebroventricular corticotropin-releasing factor in unstressed or stressed male rats. Neuroscience 51: $377-$ 390, 1992

7. Kalivas PW, Gau BA, Nemeroff CB, and Prange AJ Jr: Antinociception after microinjection of neurotensin into the central amygdaloid nucleus of the rat. Brain Res 24: 279-286, 1982

8. Al-Rodhan N, Chipkin R, and Yaksh TL: The antinociceptive effects of SCH-32615, a neutral endopeptidase (enkephalinase) inhibitor, microinjected into the periaqueductal, ventral medulla and amygdala. Brain Res 520: 123-130, 1990

9. Matsumoto N, Sato T, Yahata F, and Suzuki TA: Physiological properties of tooth pulp-driven neurons in the first somatosensory cortex (SI) of the cat. Pain 31: 249 262, 1987

10. Kawarada K, Kamata K, and Matsumoto N: Effect of electrical stimulation of the central amygdaloid nucleus on the nociceptive neuron of the cortex (SI) in the cat. Jpn J Physiol: 46: 485-490, 1996

11. Kowada K, Kawarada K, and Matsumoto N: Conditioning stimulation of the central amygdaloid nucleus inhibits the jaw-opening reflex in the cat. Jpn J Physiol 42: 443-458, 1992

12. Jasper $\mathrm{HH}$ and Ajimone-Marsan $\mathrm{C}$ : A Stereotaxic Atlas of the Diencephalon of the Cat, The National Research Council of Canada, Ottawa, 1960

13. Krettek JE and Price JL: Amygdaloid projections to subcortical structures within the basal forebrain and brainstem in the rat and cat. J Comp Neurol 178:
225-254, 1978

14. Dong WK, Chudler EH, and Martin RF: Physiological properties of intradental mechanoreceptors. Brain Res 334: 389-395, 1985

15. McGrath PA, Gracely $\mathrm{RH}$, Dubner R, and Heft NW: Non-pain and pain sensations evoked by tooth pulp stimulation. Pain 15: 377-388, 1983

16. Wilson $\mathrm{S}$ and Reid $\mathrm{KH}$ : Reflex vs behavioral responses to tooth pulp stimulation in the cat. Physiol Behav 20: 717-722, 1978

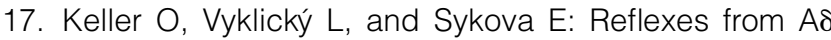
and $A \alpha$ trigeminal afferents. Brain Res 37: 330-332, 1972

18. Thexton AJ: Jaw opening and jaw closing reflexes in the cat. Brain Res 66: 425-433, 1974

19. Matsumoto N, Yahata F, Kawarada K, Kamata K, and Suzuki TA: Tooth pulp stimulation induces $c$-fos expression in the lateral habenular nucleus of the cat. NeuroReport 5: 2397-2400, 1994

20. Frenk $H$, Engel $J J r$, Ackermann RF, Shavit $Y$, and Liebeskind JC: Endogenous opioids may mediate post-ictal behavioral depression in amygdaloid-kindled rats. Brain Res 167: 435-440, 1979

21. Furui T, Harty GJ, and Yaksh TL: Studies on the effects of opioid, noradrenergic and serotonergic antagonists on the antinociceptive effects of electroconvulsive shock. Brain Res 367: 162-168, 1986

22. Oliveira MA and Prado WA: Antinociception and behavioral manifestations induced by intracerebroventricular or intra-amygdaloid administration of cholinergic agonists in the rat. Pain 57: 383-391, 1994

23. Fox RJ and Sorenson CA: Bilateral lesions of the amygdala attenuate analgesia induced by diverse environmental challenges. Brain Res 648: 215-221, 1994

24. Watkins LR, Wirtelak EP, and Maier SF: The amygdala is necessary for the expression of conditioned but not unconditioned analgesia. Behav Neurosci 107: 402-405, 1993

25. Helmstetter FJ: The amygdala is essential for the expression of conditional hypoalgesia. Behav Neurosci 3: 518-528, 1992

26. Kamal AM and Tombol T: Golgi studies on the amygdaloid nuclei of the cat. J Hirnforsch 16: 175-201, 1975

27. Gray TS and Magnuson DJ: Galanin-like immunoreactivity within amygdaloid and hypothalamic neurons that project to the midbrain central grey in the rat. Neurosci Lett 83: 264-268, 1987

28. Schwaber JS, Kapp BS, and Higgins G: The origin and extent of direct amygdaloid projections to the region of the dorsal motor nucleus of the vagus and the nucleus of the solitary tract. Neurosci Lett 20: 15-20, 1980

29. Price JL and Amaral DG: An autoradiographic study of the projections of the central nucleus of the monkey amygdala. J Neurosci 1: 1242-1259, 1981

30. Fields HL and Besson JM: Pain Modulation. Progress in Brain Research, Elsevier, Amsterdam, New York, and Oxford, Vol 77, 1988

31. Matsumoto N, Gotoh H, Sato T, and Suzuki TA: Morphine selectively suppresses the slow response of tooth pulp-driven neurons in first somatosensory cortex (SI) of the cat. Neurosci Lett 75: 55-59, 1987

32. Woda A, Azerad J, Guilbaud G, and Besson JM: Etude 
microphysiologique des projections thalamiques de la pulpe dentaire chez le chat. Brain Res 89: 193-213, 1975

33. Yokota T, Koyama N, Nishikawa $Y$, and Hasegawa A: Trigeminal nociceptive neurons in the subnucleus reticularis ventralis. II. Ascending projection. Neurosci Res 11: 18-27, 1991

34. Angus-Leppan $\mathrm{H}$, Olaussen $\mathrm{B}$, Boers $\mathrm{P}$, and Lambert GA: Convergence of afferents from superior sagittal sinus and tooth pulp on cells in the thalamus of the cat. Cephalalgia 15: 191-199, 1995

35. Rydenhag B and Roos A: Projection of tooth pulp afferents to the thalamus of the cat. II. Distribution and characteristics of single units. Exp Brain Res 64: 4958, 1986

36. Kapp BS, Supple WF Jr, and Whalen PJ: Effects of electrical stimulation of the amygdaloid central nucleus on neocortical arousal in the rabbit. Behav Neurosci 108: 81-93, 1994

37. Koyama N, Nishikawa Y, Chua AT, Iwamoto M, and Yokota T: Differential inhibitory mechanisms in VPL versus intralaminar nociceptive neurons of the cat. I. Effects of periaqueductal gray stimulation. Jpn J Physiol 45: 1005-1027, 1995

38. Chiang CY, Hu JW, and Sessle BJ: Parabrachial area and nucleus raphe magnus induced modulation of nociceptive and nonnociceptive trigeminal subnucleus caudalis neurons activated by cutaneous or deep inputs. J Neurophysiol 71: 2430-2445, 1994

39. Stock G, Schlor H, Heidt H, and Buss J: Psychomotor behaviour and cardiovascular patterns during stimula- tion of the amygdala. Pflügers Arch 376: 177-184, 1978

40. Brown MR and Gray TS: Peptide injections into the amygdala of conscious rats: effects on blood pressure, heart rate and plasma catecholamines. Regul Pept 21 : 95-106, 1988

41. Henke PG: Electrophysiological activity in the central nucleus of the amygdala: emotionality and stress ulcers in rats. Behav Neurosci 102: 77-83, 1988

42. Akil H, Madden J, Patrick RL, and Barchas JD: Concurrent analgesia and its partial reversal by naloxone. In: Opiates and Endogenous Opioid-Peptides, ed. Kosterlitz HW, Elsevier, Amsterdam, pp 63-70, 1976

43. Lewis JW, Terman GW, Nelson LR, and Liebeskind JC: Opioid and non-opioid stress analgesia. In: Stress-Induced Analgesia, ed. Tricklebank MD and Curzon Z, John Wiley \& Sons, Chichester, pp 103-133, 1984

44. Bhattacharya SK and Parmar SS: Antinociceptive effect of intracerebroventricularly administered histamine in rats. Res Commun Chem Pathol Pharmacol 49: 125-136, 1985

45. Rodgers RJ: Influence of intra-amygdaloid opiate injections on shock thresholds, tail-flick latencies and open field behaviour in rats. Brain Res 153: 211-216, 1978

46. Manning $\mathrm{BH}$ and Mayer DJ: The central nucleus of the amygdala contributes to the production of morphine antinociception in the rat tail-flick test. $J$ Neurosci 15 : 8199-8213, 1995

47. Wong $\mathrm{CL}$ : The involvement of histamine $\mathrm{H}_{2}$-receptors in restraint-induced antinociception in male mice. Meth Find Exp Clin Pharmacol 15: 351-356, 1993 